\title{
COMPARISON OF METHODS FOR MODELLING PRODUCTION FLOOR LAYOUT
}

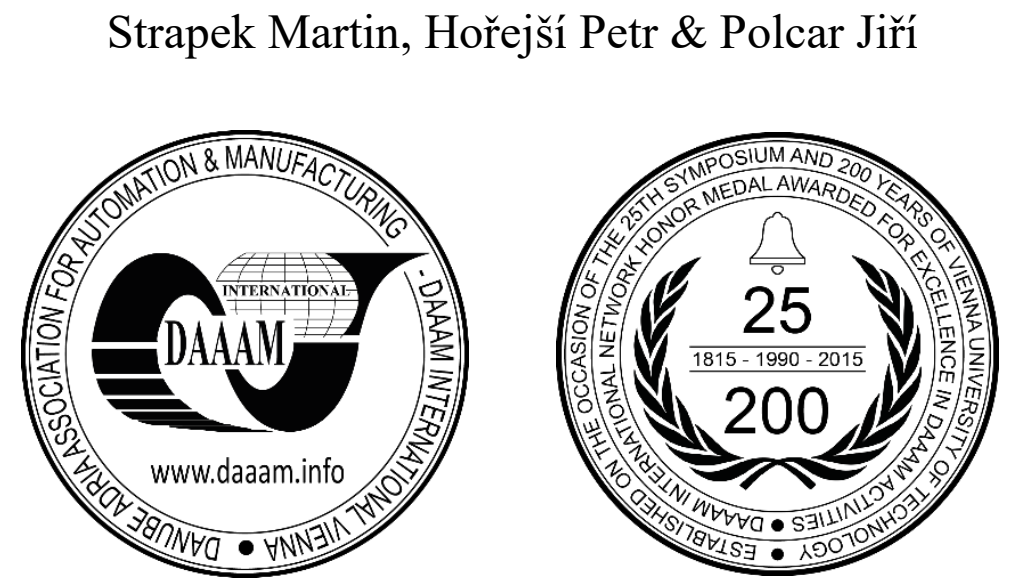

This Publication has to be referred as: Strapek, M[artin]; Horejsi, P[etr] \& Polcar, J[iri] (2017). Comparison of Methods for Modelling Production Floor Layout, Proceedings of the 28th DAAAM International Symposium, pp.0835-0844, B. Katalinic (Ed.), Published by DAAAM International, ISBN 978-3-902734-11-2, ISSN 1726-9679, Vienna, Austria DOI: $10.2507 / 28$ th.daaam.proceedings. 117

\begin{abstract}
This paper deals with comparison of two methods for production floor layout design or reengineering - conventional capturing method and 3D laser scanning. Firstly there is an analysis of the current state, where possible uses of scanning technology for production floor design or reengineering are mentioned. Then the processes of both methods are shortly described. The main part of the article is comparison of these two method. For the comparison we have used data acquired during capture of the real factory hall with both methods. The comparison is based on determined criteria - time consumption, user and hardware requirements and quality of outputs. In the conclusion, the suitability of the methods is evaluated. It also shows time consumption differences between methods and difficulty and level of requirements. In the end there are recommendations for scanning method use and for the future work.
\end{abstract}

Keywords: laser scanning; production floor modelling; factory point cloud; factory modelling

\section{Introduction}

3D laser scanning technology is no longer a new technology. This fast and precise data acquisition method is used in several areas: architecture, geodesy, archeology, criminology, show business, etc. It also penetrates into engineering, which this paper is focused on, specifically on the use of the technology for production floor planning.

The work continues on work [1], where possible methods of production floor layout modelling were described. Production floor layout [2] is one of the most important tools for production planning.

In this paper these methods are compared with the common capturing method for production floor modelling. Both methods are briefly explained. Then there are described three criteria for comparison. These were selected based on the type of expected outputs. Next, the evaluating criteria and their weights are set and then the methods are compared. The conclusion describes which method is best for each type of output. 


\section{Production floor scanning}

The first company to use 3D laser scanning for layout planning was Volvo. At that time (1996-1997), the available technology was limited. But since then, many experiments using the technology in different areas have been made. Production floor layout modelling [3], visualization and problem solving in production [4] and connecting scanned models into a simulated environment [5] have been developed for the engineering field.

Data acquisition and data processing are described by [6], and for production floors specifically by [1]. During the scanning process, the spatial coordinates of a scanned object are acquired and these are then converted into digital form, a 'point cloud'. An example of a point cloud is given in Fig. 1. Due to the very high performance of scanning devices, it is more important to focus on processing the acquired data. Work with these data is sometimes very complicated, especially when we want to generate a full 3D model from a point cloud model. This issue is dealt with for example by [7] and [8]. Other possible uses of algorithmization are being worked on [9]. This algorithm is used in the data processing method [1] for rapid creation of a 2D production floor layout from a point cloud model. Parts of scanned production floors can be also used as models for augmented reality systems [10].

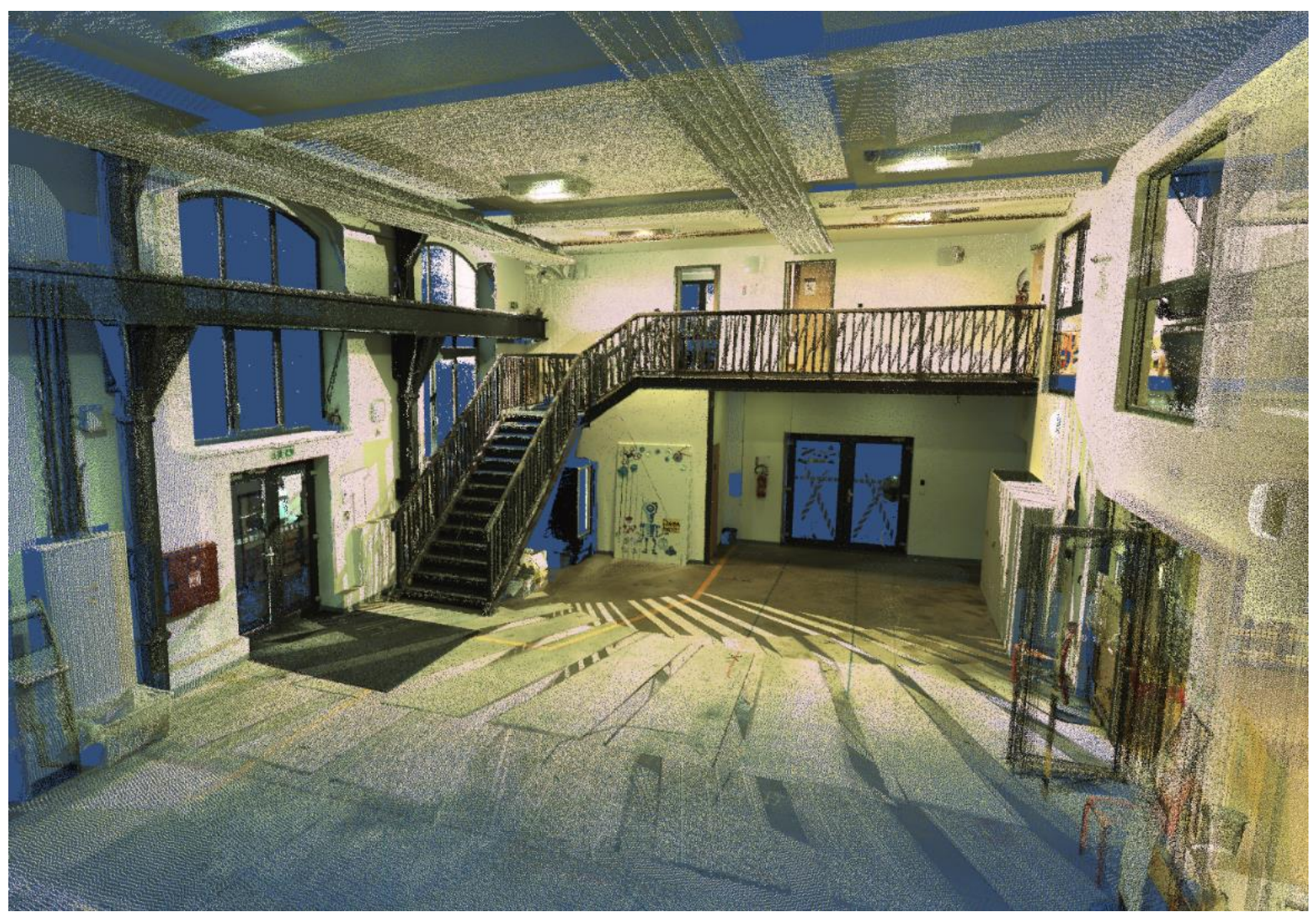

Fig. 1. Example of building point cloud

When acquiring data with laser scanning it is necessary to use a scanning device. As was mentioned, performance of these devices is now at a much higher level than older models. The main limiting parameter of a scanner is its scanning speed. The highest scanning speeds these days are about 1000000 points per second. It is probably the highest possible speed. Even higher speeds are not necessary, because they would require new technologies, and the time savings would be insignificant compared to the present devices.

Instead of increasing speed, the producers of these devices are focused on more tailor made scanners. A great example is the BLK 360 [11]. This scanner has 360000 pts/s speed, which is definitely enough for high quality point cloud acquisition in a very short time. This speed is more than enough for production floor scanning, so it would be useless to increase the cost of a faster scanner. On top of that, BLK360 excels with its dimensions, which reduce the problems with scanner positioning in a limited production floor space.

\section{Methodology}

The following sections describe how the use of scanners effects production floor layout modelling. The times of individual parts of the process will be shown, as will other criteria. These values will be compared with the basic method for production floor modelling and recommendations will be made for when to use a specific method. 


\subsection{Conventional capturing method}

For classic capturing and modelling, common measuring tools (laser meter, camera, machine and equipment sketches) are used. For the classic method, employees must capture the dimensions of the basic factory hall, machines, equipment and objects in the factory and their relative positions. Based on these acquired data and use of special software, a layout model can be made. It is necessary to create models of machines, equipment and objects, then the factory hall itself and place all the objects exactly as in the real state.

\subsection{Laser scanning method}

For the scanning method we used a 3D laser scanner (in our case Leica C5). In this method, the whole factory hall (including all objects) was scanned. Then the acquired data were loaded into special software, where a complete 3D model was created. This model can be used for further processing as described in section Production floor scanning. The technical parameters of our scanning devices are on [12]. The most important parameter is scanning speed, which is 25000 points per second (pts/s) in our case.

\subsection{Reference model and outputs}

A real factory was chosen to compare the methods. The factory was first measured and modelled using the classic method. Next we tried to acquire the same (or similar) output using 3D laser scanning. The time between both captures was about one year. Each capture was done by two different teams of employees. For both methods, two employees were used. In each group, we used experts in the specific method.

Both of these methods were tested in three main categories, which were determined based on the required output:

- $2 \mathrm{D}$ block scheme layout (layout modelled on the level of workplaces or parts of the production)

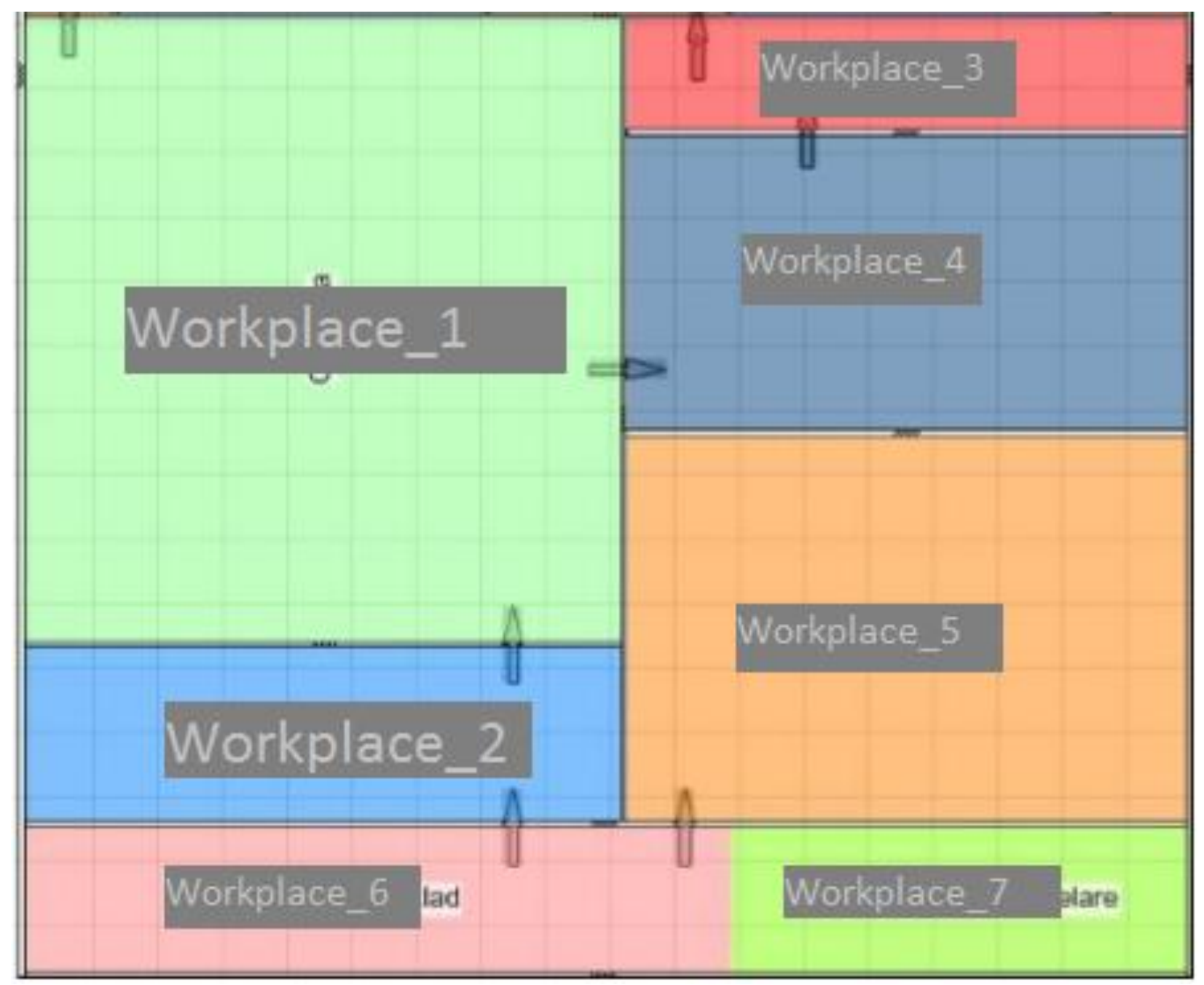

Fig. 2. Example of 2D block scheme layout 
- $\quad$ 2D machine layout (layout modelled on the level of individual machines and equipment)

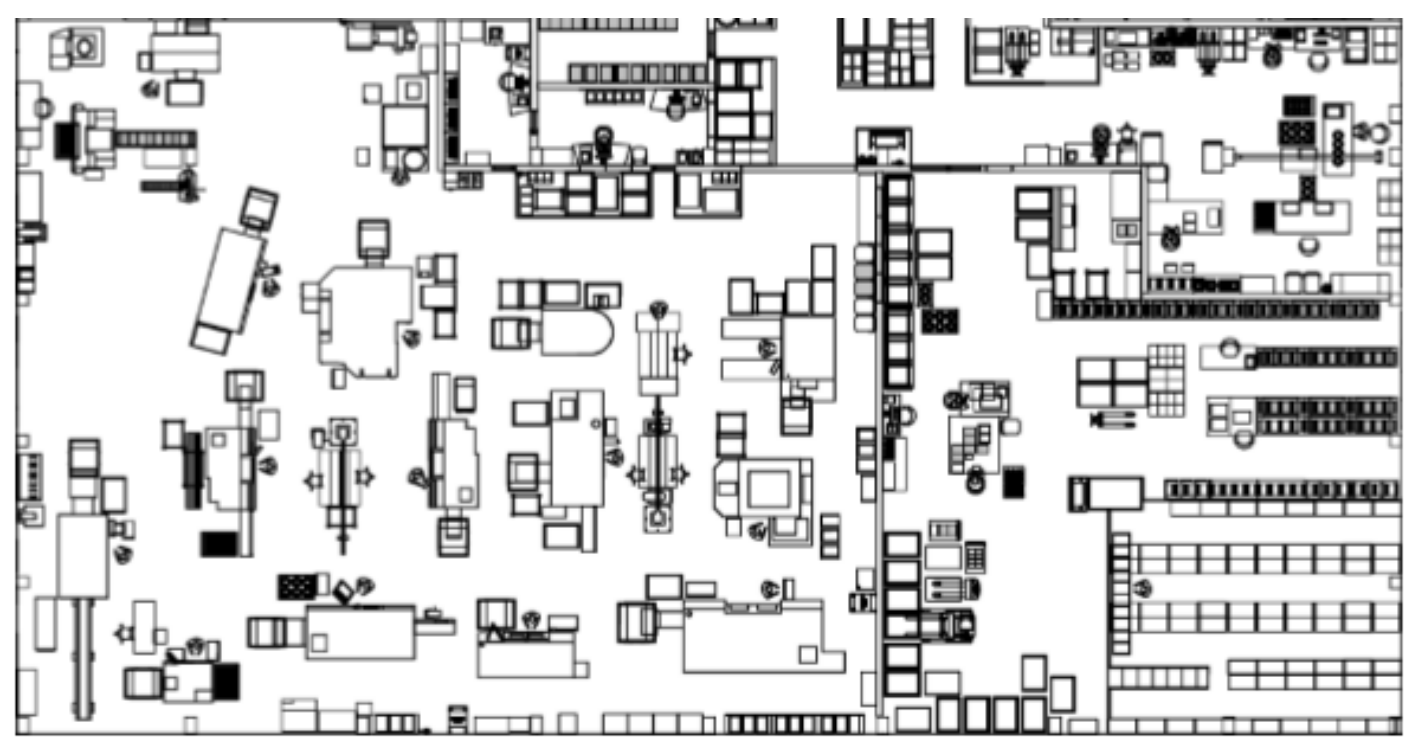

Fig. 3. Example of 2D machine layout

- $\quad 3 \mathrm{D}$ machine layout (layout modelled on the level of individual machines and equipment and visualized in 3D)

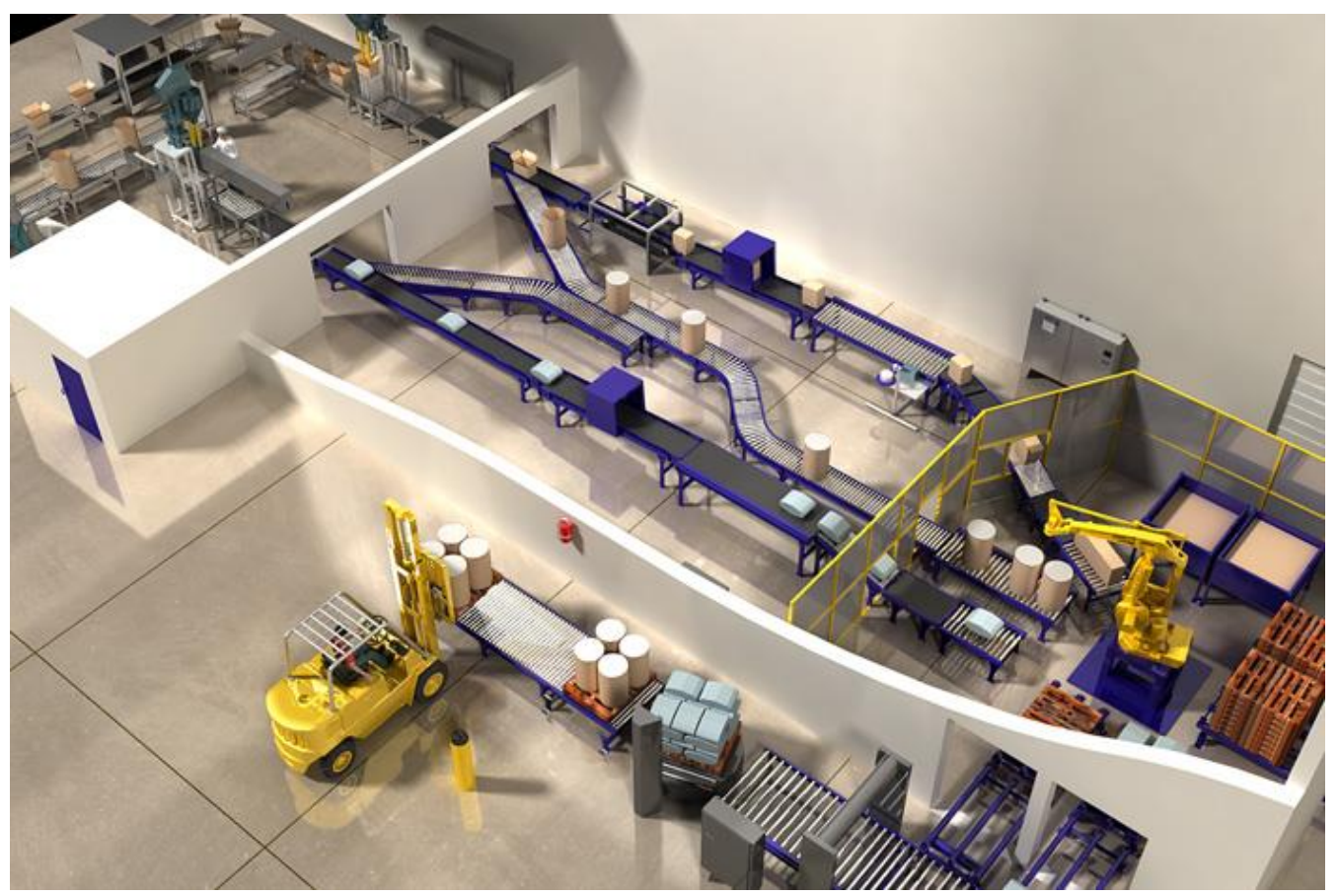

Fig. 4. Example of 3D machine layout

The effort was to create models that have a similar use for further processing. Currently, our most used software for layout modelling is VisTable software [13]. This software is very user friendly, cheap and it contains the analyzing tools which are required for layouts. We wanted to create outputs from the scanned models that have the same possibilities for analysis as the outputs from the classic method.

\subsection{Criteria}

The evaluation criteria were selected for each category. These criteria evaluate the whole process of output creation and its quality:

a) Data acquisition time 
These times were taken during data acquisition in both methods. As already mentioned, the selected factory was first captured using the classic method, and then it was scanned using the Leica C5 laser scanner.

For comparison, we estimated the final times for a more powerful scanning device (for example, Leica BLK360). The time duration is considered from the start of measurement to the acquisition of the last dimension. Times needed for acquiring missing dimensions are also counted in this time.

\section{b) Output development time}

This time was measured from the first processing of the acquired data to the end of creation of the complete model. In the classic method this is the total of:

- Sum of times needed for creating models of machines, equipment and objects in CAD modelling software (times are converted to one man work)

- $\quad$ Time needed for creating layout in visTable software or Autodesk Factory Design (times for creating model in both types of software are similar)

The classic method output was a layout in visTable format. The output is a layout which is completely ready for subsequent analysis.

In the scanning method, the output creation time is the total of:

- $\quad$ Time needed for transferring scanned data into computer (because scanned data has very big volume, it was necessary to count this time)

- $\quad$ Time needed for cleaning data (removal of unnecessary point clouds)

- $\quad$ Time needed for data registration (registration = assembling individual scans into one complete model)

c) User requirements

User qualifications and skills must be at a level such that s/he can produce the required output at the required quality using the appropriate method. This criterion was set based on the quantity of operations, software and equipment which the user must handle during the process of data acquisition and output creation.

In the classic method, the user must handle a laser meter and sketching. These are activities which do not require any special skills. For layout creation, the user must handle visTable software and modelling CAD software. These activities usually go in parallel, so only knowledge of one particular type of software is needed for the user.

In the scanning method the user must handle the scanning device. Basic training for using a scanning device can take $1-$ 2 days. The user must handle work with software for scanned data processing (Leica Cyclone in our case). Basic training and mastering skills with the software can take 1 day. For further data processing (AutoCAD, visTable, Inventor) the user must also know how to work with this software. Due to the connection of all activities during data processing, it is appropriate for one user to master them.

The classic method requires the knowledge of two types of software for a given methodology. The scanning method requires working with point clouds in this software and also requires the knowledge of scanned data processing software. The scale of evaluating user requirements was set based on these criteria. The scale is from low requirements to very high requirements.

\section{d) Hardware requirements}

In hardware requirements, the computer equipment for creating smooth outputs was evaluated. The classic method does not require any special equipment. The recommended requirements for visTable software [13] and for AutoCAD software [14] meet the performance of commonly used computers.

For the scanning method, even for meeting the Leica Cyclone requirements [15], there is a need for a high performance computer. When working with large projects, like factory halls, there is a need for the computer to process a big volume of data (sometimes GBs files).

\section{e) Quality}

Match with the real object, visualization and clarity of model is evaluated in the quality criterion. In the classic method, we only model things which affect the production system- the layout model is clear for understanding the process.

The scanning method captures the whole production system including objects unusable in production floor analyses. However, these objects can be used for other analyses (pipeline, distribution of electricity, air conditioning, etc.).

\section{Method comparison}

The multi-criteria decision making method was used to compare the methods. For the above-mentioned criteria, we set criteria weights according to the Table 1 . We used the pair comparison method for determination of weights. 
The most important criteria are time values, then hardware and the user requirements. Quality has the lowest weight because this is mainly the visualization of the output. Dimension matches with real objects are within the available norm for all methods.

\begin{tabular}{|c|c|c|c|c|c|c|}
\hline Criterion & $\begin{array}{c}\text { Data } \\
\text { acquisition } \\
\text { time (h) }\end{array}$ & $\begin{array}{c}\text { Output } \\
\text { creation time } \\
\text { (h) }\end{array}$ & $\begin{array}{c}\text { User } \\
\text { requirements }\end{array}$ & $\begin{array}{c}\text { Hardware } \\
\text { requirements }\end{array}$ & Quality & SUM \\
\hline Weight & 0.3 & 0.3 & 0.15 & 0.175 & 0.075 & 1 \\
\hline
\end{tabular}

Table 1. Criteria and its weigths

Input values for both methods are given in the Table 2., 3. and 4. Numerical values were set based on the measured data, others were set according to 3.4 Criteria. We compared the classic method, the scanning method using a Leica C5 and a method using a BLK360 device (time values were converted using the scanning speed parameter).

\begin{tabular}{|c|c|c|c|c|c|}
\hline Output/Criterion & $\begin{array}{c}\text { Data } \\
\text { acquisition } \\
\text { time }(\mathrm{h})\end{array}$ & $\begin{array}{c}\text { Output creation } \\
\text { time (h) }\end{array}$ & $\begin{array}{c}\text { User } \\
\text { requirements }\end{array}$ & $\begin{array}{c}\text { Hardware } \\
\text { requirements }\end{array}$ & Quality \\
\hline 2D block scheme & 14 & 2 & Low & Low & High \\
\hline $\begin{array}{c}\text { 2D machine } \\
\text { layout }\end{array}$ & 31 & 63 & Medium & Low & High \\
\hline $\begin{array}{c}\text { 3D machine } \\
\text { layout }\end{array}$ & 34 & 63 & Medium & Medium & High \\
\hline
\end{tabular}

Table 2. Input data for classic method

\begin{tabular}{|c|c|c|c|c|c|}
\hline Output/Criterion & $\begin{array}{c}\text { Data } \\
\text { acquisition } \\
\text { time }(\mathrm{h})\end{array}$ & $\begin{array}{c}\text { Output creation } \\
\text { time }(\mathrm{h})\end{array}$ & $\begin{array}{c}\text { User } \\
\text { requirements }\end{array}$ & $\begin{array}{c}\text { Hardware } \\
\text { requirements }\end{array}$ & Quality \\
\hline 2D block scheme & 8 & 4.5 & High & Very high & Very high \\
\hline $\begin{array}{c}\text { 2D machine } \\
\text { layout }\end{array}$ & 15 & 5 & High & Very high & Very high \\
\hline $\begin{array}{c}\text { 3D machine } \\
\text { layout }\end{array}$ & 15 & 6 & High & Very high & Very high \\
\hline
\end{tabular}

Table 3. Input data for laser scanning method using C5 device

\begin{tabular}{|c|c|c|c|c|c|}
\hline Output/Criterion & $\begin{array}{c}\text { Data } \\
\text { acquisition } \\
\text { time }(\mathrm{h})\end{array}$ & $\begin{array}{c}\text { Output creation } \\
\text { time }(\mathrm{h})\end{array}$ & $\begin{array}{c}\text { User } \\
\text { requirements }\end{array}$ & $\begin{array}{c}\text { Hardware } \\
\text { requirements }\end{array}$ & Quality \\
\hline 2D block scheme & 2 & 4.5 & High & Very high & Very high \\
\hline $\begin{array}{c}\text { 2D machine } \\
\text { layout }\end{array}$ & 3 & 5 & High & Very high & Very high \\
\hline $\begin{array}{c}\text { 3D machine } \\
\text { layout }\end{array}$ & 3 & 6 & High & Very high & Very high \\
\hline
\end{tabular}

Table 4. Input data for laser scanning method using BLK360 device

Next, the criteria values were scored $1-5(1-$ the worst, 5 - the best), followed by multiplying by criteria weights. The weighted criterion sum represents the suitability of using individual methods.

\subsection{D block scheme layout}

According to Table 5. And Fig. 5. we can see that for creating a simple block scheme layout, it is most appropriate to use the classic method. Even though Fig. 6 shows that data acquisition time and output creation time are a little shorter for the scanning method, according Fig. 7, using the scanning method is unnecessarily complicated and difficult. Because the graphs for other criteria comparison are almost the same, we have merged them into one graph. 


\begin{tabular}{|c|c|c|c|c|c|c|}
\hline Method/Criterion & $\begin{array}{c}\text { Data } \\
\text { acquisition } \\
\text { time }(\mathrm{h})\end{array}$ & $\begin{array}{c}\text { Output } \\
\text { creation time } \\
(\mathrm{h})\end{array}$ & $\begin{array}{c}\text { User } \\
\text { requirements }\end{array}$ & $\begin{array}{c}\text { Hardware } \\
\text { requirements }\end{array}$ & Quality & SUM \\
\hline Classic & $1^{*} 0.3$ & $5^{*} .03$ & $5^{*} 0.15$ & $5^{*} 0.175$ & $3^{*} 0.075$ & 3.65 \\
\hline Leica C5 scanner & $3^{*} 0.3$ & $3^{*} .03$ & $1^{*} .015$ & $1^{*} 0.175$ & $5^{*} 0.075$ & 2.5 \\
\hline BLK360 scanner & $5^{*} 0.3$ & $3^{*} .03$ & $1^{*} 0.15$ & $1 * 0.175$ & $5^{*} 0.075$ & 3.1 \\
\hline
\end{tabular}

Table 5. 2D block scheme layout evaluating

2D block scheme layout evaluation

4

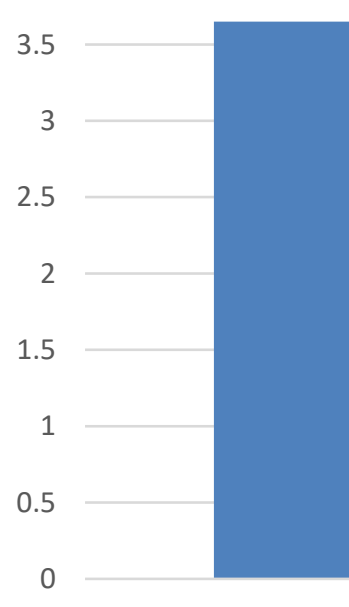

Classic
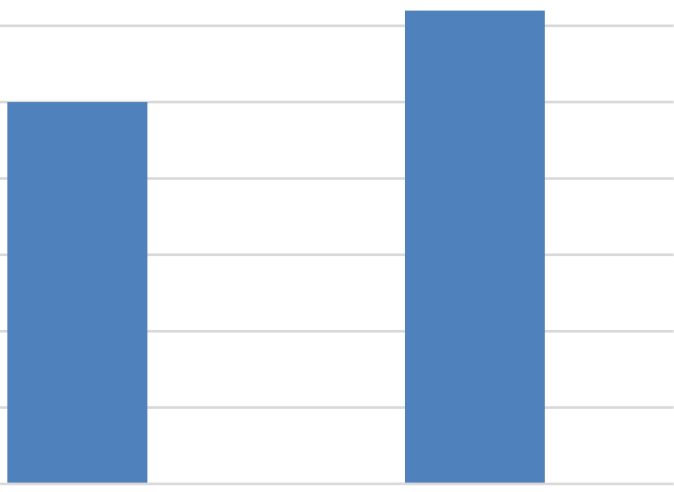

Scan C5

Scan BLK360

Fig. 5. Comparison of methods for 2D block scheme layout modelling

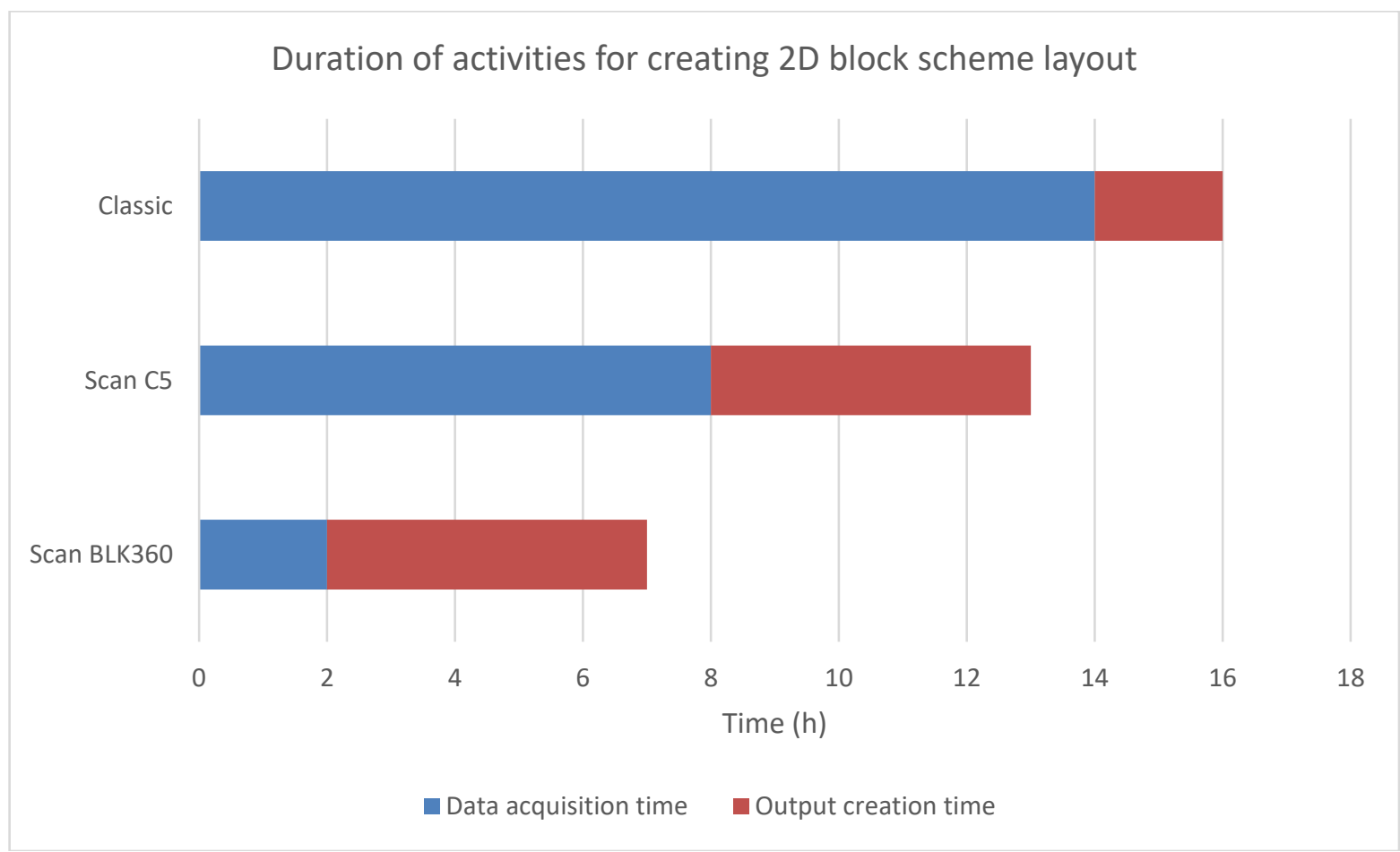

Fig. 6. Activities duration comparison for 2D block scheme layout 


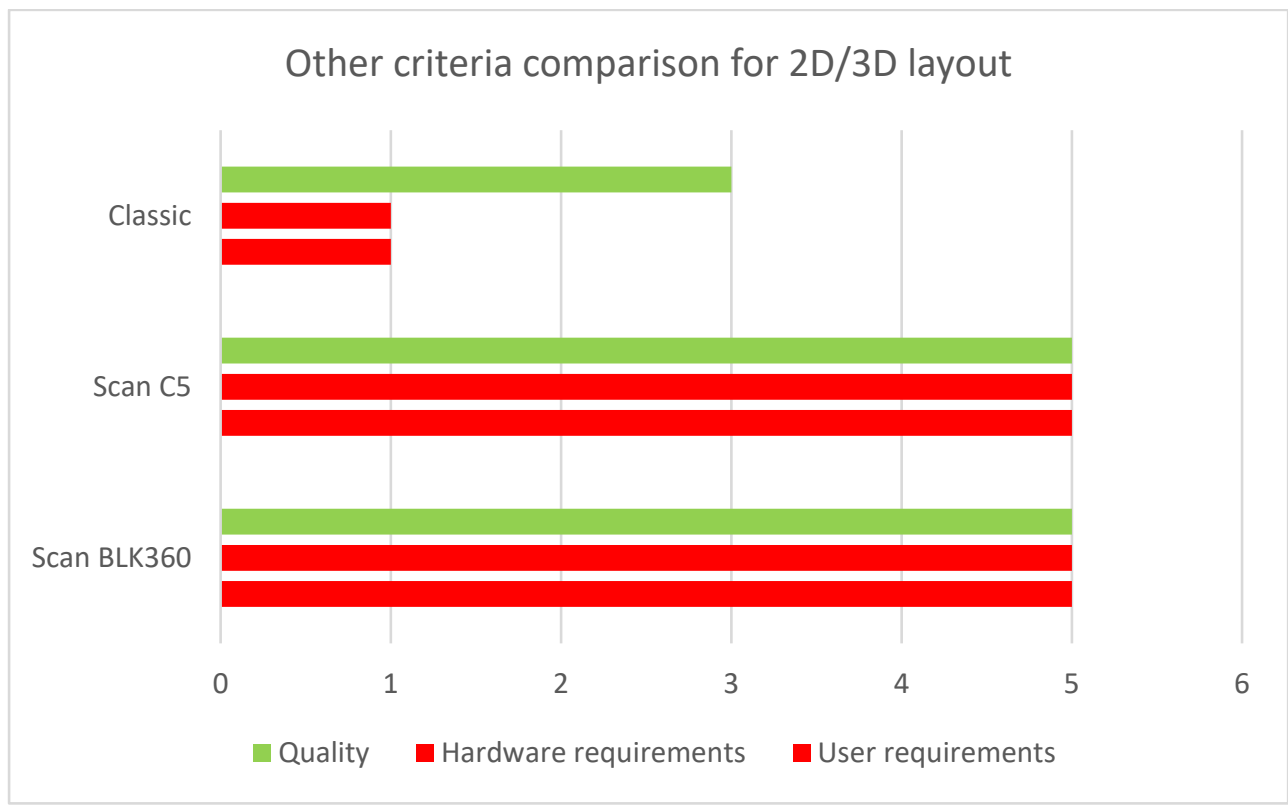

Fig. 7. Criteria comparison for 2D/3D layout

\subsection{D/3D machine layout}

Also, because time values and other criteria values for both of these outputs are very similar, we have merged them into one category. For these outputs according to Table 6.and Fig. 8. it is preferable to use scanning methods. The best choice by far is the device BLK 360. Time savings are approximately 5 times shorter, which compensates for the higher requirements of the method.

\begin{tabular}{|c|c|c|c|c|c|c|}
\hline Method/Criterion & $\begin{array}{c}\text { Data } \\
\text { acquisition } \\
\text { time (h) }\end{array}$ & $\begin{array}{c}\text { Output } \\
\text { creation time } \\
(\mathrm{h})\end{array}$ & $\begin{array}{c}\text { User } \\
\text { requirements }\end{array}$ & $\begin{array}{c}\text { Hardware } \\
\text { requirements }\end{array}$ & Quality & SUM \\
\hline Classic & $1^{*} 0.3$ & $1^{*} .03$ & $5^{*} 0.15$ & $5^{*} 0.175$ & $4^{*} 0.075$ & 2.525 \\
\hline Leica C5 scanner & $3^{*} 0.3$ & $5^{*} .03$ & $1^{*} .015$ & $1^{*} 0.175$ & $5^{*} 0.075$ & 3.1 \\
\hline BLK360 scanner & $5^{*} 0.3$ & $5^{*} .03$ & $1^{*} 0.15$ & $1^{*} 0.175$ & $5^{*} 0.075$ & 3.7 \\
\hline
\end{tabular}

Table 6.2D/3D machine layout evaluating

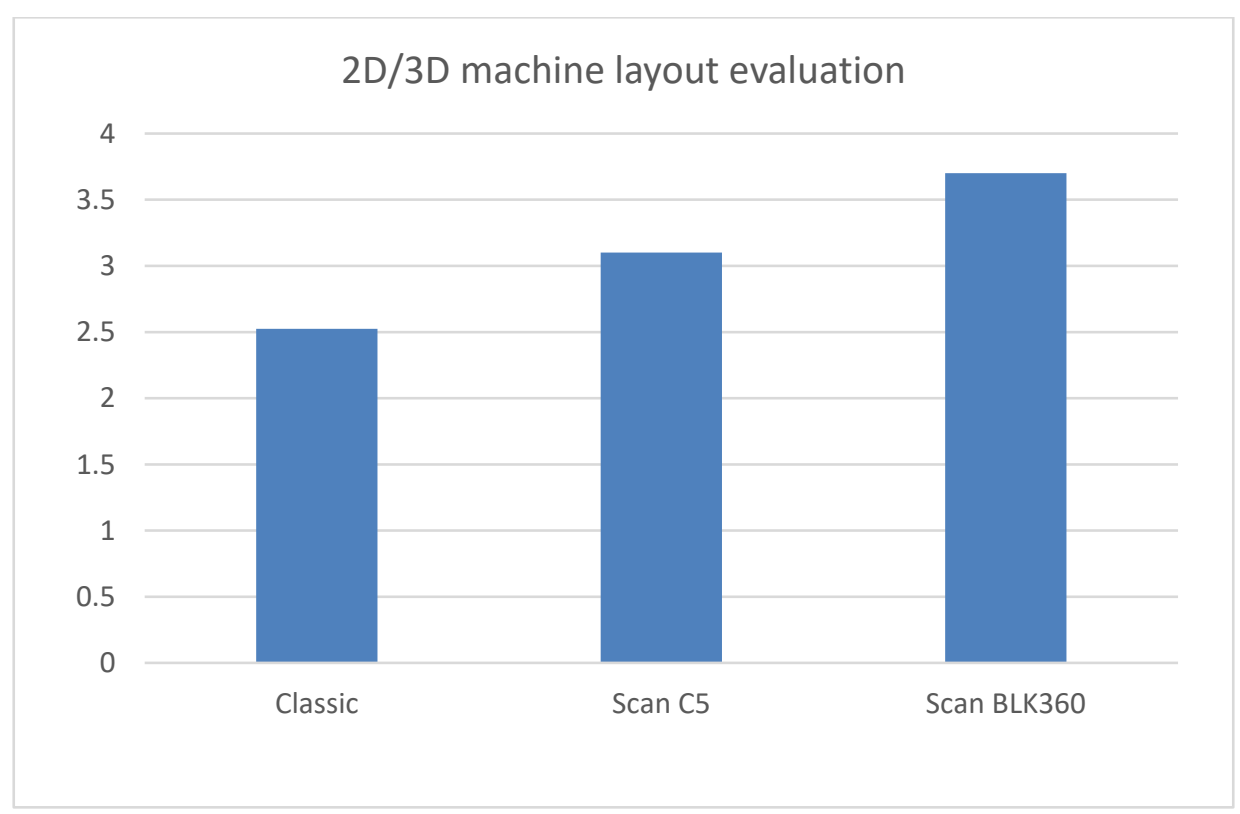

Fig. 8. Comparison of methods for 2D/3D machine layout modelling 


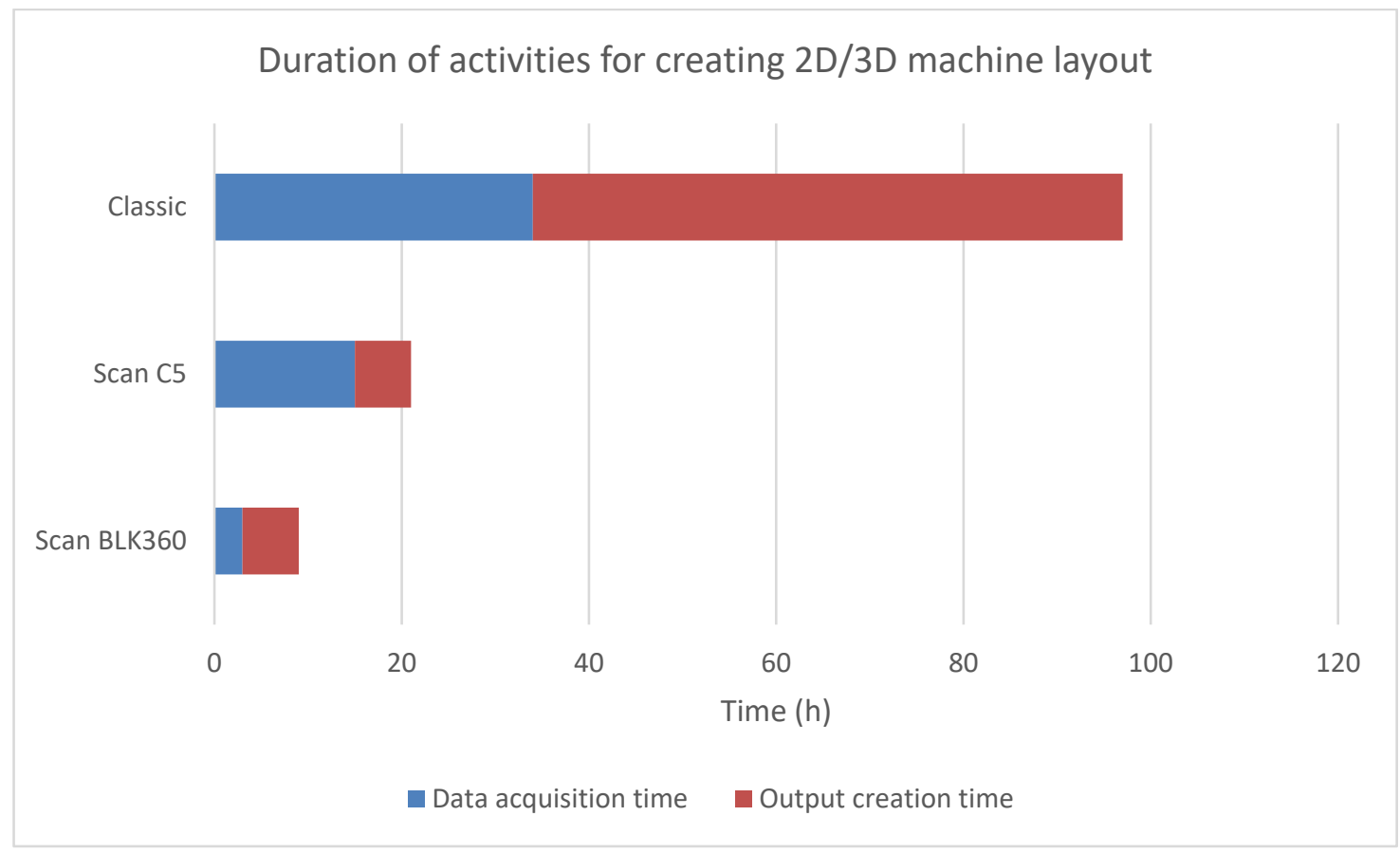

Fig. 9. Activities duration comparison for 2D/3D machine layout

\section{Evaluation of results, recommendations, future work}

The main purpose of this work was to compare efficiency of two methods for production floor layout modelling. For the comparison, the real factory was chosen and both methods were tested. During testing, we were acquiring data for comparison - time values, requirements for methods and quality of outputs.

Based on the results we can say that for creating a block scheme it is more suitable to use the classic measuring method.

Even though creating a layout using this method takes longer, using the scanning method would be too complicated. We would have to use complicated processes (scanning, data transformation, clearing data and creating output) and the time savings are not that big. When using the scanning method for machine layout capture, especially 3D, the time saving is already big enough that the method is more suitable.

Economically, purchase of scanning equipment and necessary hardware is recommended for repeated use of the scanning device. It is more suitable for bigger companies with a large number of factories, or for companies which offer scanning as their service. For scanning a production floor only once it is better to outsource the services of a company which already has the needed equipment.

Due to the falling price of scanners with parameters suitable for production floor measurement, it is probable that more companies will use this method for capturing their layouts. With the increasing amount of scanned data used, new opportunities in algorithmization can arise, for example, an algorithm for automatically generating proper primitives and library models from point clouds.

Because we have found scanning technology useful for production floor modelling, we will focus on developing algorithms for modelling simplification in the future work. Also we research possibilities for processing scanned data with the use of virtual and augmented reality devices like motion tracking systems.

\section{Acknowledgement}

This paper was prepared with the support of the Internal Science Foundation of the University of West Bohemia SGS2015-065.

\section{References}

[1] Strapek, M., Hořejší, P., Polcar, J. (2016) 3D Laser Scanned Data Processing Possibilities for Production Floors Models. In Proceedings of the 28th International Business Information Management Association Conference. Norristown: International Business Information Management Association, 2016. pp. 2920-2930. ISBN: 978-09860419-8-3

[2] Chen, D., Shariatzadeh, N. (2012), „Software Eraluation Criteria for Rapid Factory Layout Planning, Design and Simulation“ at 45 CIRP Conference on Manufacturing Systems, ISBN 978-1-627-48512-8 Stockholm. 
[3] E. Shellshear, R. Berlin, J. S. Carlson (2015), "Maximizing Smart Factory Systems by Incrementally Updating Point Clouds”, IEEE Computer Graphic and Applications, sv. XXXV, č. 2 ISSN 0272-1716, pp. 62-69.

[4] Lindskog E., Berglund J., Vallhagen J., Johansson B. (2014), „Lean Based Problem Solving using 3D Laser Scanned Visualizations of Production Systems“ at International Journal of Engineering Science and Innovative Technology, ISSN: 2319-5967.

[5] Lindskog E., Berglund J., Vallhagen J., Berlin R., Johansson B. (2012), „Combining Point Cloud Technologies with Discrete Event Simulation“ at 2012 Winter Simulation Conference, ISBN: 978-1-467-34781-5 Berlin

[6] Bi, Z.M., Wang, L. (2010), Advances in 3D data acquisition and processing for industrial applications. From: Robotics and Computer-Integrated Manufacturing 26. March 2010. ISSN: 0736-5845

[7] Xiong, X., Adan, A., Akinci, B., Huber, B. „Automatic creation of semantically rich 3D building models from laser scanner data" at Automation in Construction. ISSN: 0926-5805. [2013]

[8] Tang, P., Huber, D., Akinci, B., Lipman, R., Lytle, A. „Automatic reconstruction of as-built building information models from laser-scanned point clouds: A review of related techniques“ at Automation in Construction. ISSN: 0926-5805. [2010].

[9] Polcar, J., Gregor, M., Hořejší, P., Kopeček, P. Projection of LiDAR Point Cloud Slices to Raster Images as 3D Modelling Underlays. In Innovation Management and Sustainable Economic Competitive Advantage: From Regional Development to Global Growth. Madrid: International Business Information Management Association, 2015. s. 2220-2226. ISBN: 978-0-9860419-5-2

[10] Hořejší, P., (2015), Augmented Reality Systems for Virtual Training of Parts Assembly in Procedia Engineering, Volume 100 - 2015, ISSN 1877-7058, edit Katalinic, P., pp. 699-706, Zadar, Croatia.

[11] https://lasers.leica-geosystems.com (2017). Leica BLK360 Product specification, Accessed on: 2017-06-21

[12] https://gefos-leica.cz (2017). Leica C5 Product specifications, Accessed on: 2017-06-21

[13] http://cloud.vistable.de (2017). Systemvoraussetzungen-Requirements, Accessed on: 2017-06-20

[14] https://knowledge.autodesk.com (2017). System requirements for AutoCAD 2017, Accessed on: 2017-06-20

[15] https://hds.leica-geosystems.com (2017). Leica Cyclone datasheet, Accessed on: 2017-06-19 